\title{
COVID-19 und Psoriasis-Patienten
}

Auf dem 30. Kongress der EADV (Europäische Akademie für Dermatologie und Venereologie) wurden Real-World-Daten vorgestellt, die sich mit der Biologika-Therapie und der zögerlichen Impfbereitschaft von Psoriasis-Patienten befassen. Die Ergebnisse zweier Studien tragen dazu bei, das Verständnis für die Sicherheit von Biologika-Therapien bei Psoriasis und die Gründe für diese zögerliche Haltung gegenüber COVID-19-Impfstoffen bei Psoriasis-Patienten zu verbessern.

Spanische Forscher haben die Einstellung zur COVID-19-Impfung bei mit Biologika behandelten Patienten mit Psoriasis und/oder Psoriasis-Arthritis untersucht [1]. Die Real-WorldDaten wurden über soziale Medien erhoben, um die Einschränkungen herkömmlicher Krankenhausumfragen zu minimieren, bei denen nur Daten von motivierten Patienten erfasst werden, die durch die Anwesenheit eines Arztes beeinflusst sein könnten. Zwischen Januar und März 2021 wurden 10.922 Social-Media-Beiträge von Patienten in den USA, dem Vereinigten Königreich, Frankreich, Deutschland und Spanien anhand von vordefinierten Schlüsselwörtern identifiziert. Aus diesen wurden dann 624 Beiträge herausgefiltert, die manuell analysiert wurden.

\section{Sicherheitsbedenken}

Die Ergebnisse deuten darauf hin, dass das Zögern der Patienten betreffend COVID-19-Impfstoffe auf Sicherheitsbedenken und die Sorge vor einer Verschlimmerung ihrer Grunderkrankung zurückzuführen ist $(n=344)$. Hauptfaktoren für diese Wahrnehmung waren mögliche Nebenwirkungen nach der Impfung, die Auswirkungen auf ihre Autoimmunerkrankung und fehlende Studiendaten.

Darüber hinaus hatten die Patienten keine Informationen über die Wechselwirkung der COVID-19-Impfstoffe mit der Biologika-Therapie und wussten nicht, welche Wirkung die Impfung bei immungeschwächten Patienten hat.

Dr. Álvaro González-Cantero von der Abteilung für Dermatologie des Hospital Universitario Ramon Y Cajal in Madrid, Spanien, sagte: „Mit Blick auf die Zukunft wissen wir, dass wir diese Ergebnisse berücksichtigen und die Patienten über die Bedeutung, Sicherheit und Wirksamkeit der COVID-19-Impfung aufklären müssen."

\section{Biologika-Therapien und Atem- wegsinfektionen}

Biologika-Behandlungen der Schuppenflechte sind häufig mit einem erhöhten Infektionsrisiko verbunden. In einer zweiten Real-World-Studie untersuchten Forscher in den Niederlanden die unterschiedlichen Auswirkungen von Biologika-Therapien auf das Risiko von Atemwegsinfektionen (RTI) und schweren Infektionen (SI), einschließlich COVID-19, um festzustellen, ob hier ein Zusammenhang besteht [2].

Es wurde eine Praxiskohorte von 714 Psoriasis-Patienten mit 1325 Behandlungsepisoden aus dem BioCAPTURE-Register analysiert, wobei 2224 RTI und 63 SI gemeldet wurden, aber nur 1,3\% der RTI als schwerwiegend gemeldet wurden.

Die Analyse ergab kein unterschiedliches Risiko für RTI zwischen den eingeschlossenen Biologika Adalimumab, Etanercept, Infliximab, Ustekinumab, Secukinumab, Ixekizumab und Guselkumab und auch keinen Zusammenhang mit schweren Infektionen. Was die SARS-CoV-2-Infektionen betrifft, so lag die rohe Inzidenzrate im Jahr 2020 in einem einzigen BioCAPTUREZentrum bei 3,8 (95\% Cl: 2,2-6,1) pro 100 Patientenjahre.

„Unsere Analyse zeigt keine Unterschiede beim Risiko von Atemwegsinfektionen zwischen Biologika, einschließlich der neueren IL-17- und
IL-23-Inhibitoren, in einer prospektiven Kohorte von Psoriasis-Patienten. Darüber hinaus deuten unsere vorläufigen Ergebnisse darauf hin, dass Biologika-Behandlungen keinen Einfluss auf die Anfälligkeit von Psoriasis-Patienten für COVID-19-Infektionen haben", so Dr. Lara van der Schoot von der Abteilung für Dermatologie am Radboud University Medical Center, Nijmegen, Niederlande, und Hauptautorin der Studie.

\section{Literatur}

1. González-Cantero A et al (2021) Understanding real-world experiences and the impact of COVID-19 vaccination on the associated use of biologics in patients with psoriasis and psoriatic arthritis through social media monitoring. Abstract no 181 submitted to EADV 30th Congress 2021

2. van der Schoot L et al (2021) Risk of serious and respiratory tract infections in psoriasis patients treated with biologics: results from the Biocapture registry. Abstract no. 1161 submitted to EADV 30th Congress 2021

Hinweis des Verlags. Der Verlag bleibt in Hinblick auf geografische Zuordnungen und Gebietsbezeichnungen in veröffentlichten Karten und Institutsadressen neutral.

hautnah $2021 \cdot 20: 182-183$ https://doi.org/10.1007/s12326-02100485-z

(c) Springer-Verlag GmbH Austria, ein Teil von Springer Nature 2021 
Hier steht eine Anzeige.

Springer 